\title{
UMA FERRAMENTA DE ANÁLISE SUBJECTIVA DAS INSTITUIÇÕES QUE TRANSFEREM TECNOLOGIA
}

\author{
A tool for subjective analysis of institutions that \\ transfer technology \\ David Nunes Resende* \\ Henrique Manuel Moreira Diz** \\ Dálcio Roberto dos Reis***
}

\section{Resumo}

O objectivo deste artigo é apresentar uma proposta de metodologia para análise subjectiva de processos de transferência de tecnologia em instituições de I\&D. Procuramos identificar "bottlenecks" naqueles processos a partir do mapeamento destes numa estrutura de facilitadores montada com base nas práticas actuais observadas em instituições de I\&D norte-americanas e europeias.

Conseguimos compilar 275 regras referentes a 54 facilitadores distribuídos em 7 grupos.

Esta metodologia foi montada de forma a gerar como saída um relatório de análise e recomendações capaz de optimizar os processos da instituição de forma hierarquizada.

Os procedimentos metodológicos consistiram, fundamentalmente, por análise documental e observação participativa.

* Professor da Universidade de Aveiro - Escola Superior de Tecnologia e Gestão de Águeda. Email: david@ua.pt

** Professor da Universidade de Aveiro. Email: hdiz@ua.pt

*** Professor do Programa de Pós-graduação em Tecnologia da Universidade Tecnológica Federal do Paraná. Email: dalcio@utfpr.edu.br 
uma ferramenta de análise...

Palavras-chave: Transferência de tecnologia. Investigação. Desenvolvimento. Universidade. Academia.

\section{Abstract}

The objective of this article is to present a methodology proposal to subjective analysis of processes in Technology transfer of R\&D institutions.

We are looking for to identify "bottlenecks" in those processes based on mapping them in our structure of "facilitators". This structure was mounted based in the actual practices observed in Europe and North America institutions of R\&D.

One of the most important result of our research was the compilation of 275 rules referring to 54 facilitator distributed in 7 groups.

This methodology was created so that the output was in form of a report, the Analysis Report. This report carry out the processes reengineered and recommendations capable of optimize the most important processes in hierarchical form.

The methodological procedures consisted, basically, in documental analysis and participant observation

Keywords: Technology transfer. Research. Development. University. Academy.

\section{INTRODUÇÃO}

Nos dias que correm é cada vez mais notória a importância das tecnologias. A dita "Vantagem Competitiva" tem como um dos seus grandes propulsores a tecnologia e o conhecimento. O grande progresso verificado em âmbito mundial, impulsionado em parte substancial pelas novas tecnologias, entre estas aquelas ligadas ao recente mundo "digital" e outras empurradas pelo avanço da integração dos circuitos electrónicos, estão a proporcionar desenvolvimentos em todas as áreas da actividade humana, quer sejam ligadas aos setores empresarial, económico ou social.

Por isso, e com a elevada taxa de mudança tecnológica imprimida pela concorrência mundial, as empresas vêm-se obrigadas, a focar as suas atenções nos aspectos tecnológicos das suas actividades. 
Na Europa as dificuldades decorrentes do Mercado Único Europeu, que aumentaram a concorrência com a alta competitividade das empresas estrangeiras que agora actuam livremente no mesmo espaço, acrescido do alargamento aos países de leste, alguns com níveis de escolaridade elevados e cuja mão-de-obra ainda é barata, tem levado as empresas nacionais a uma maior preocupação pelos aspectos de melhoria dos seus parques tecnológicos. Da mesma forma a voltarem mais atenção sobre os mecanismos internos que thes proporcionem acesso, de forma mais simples/rápida (eficiente) e barata ao conhecimento e às tecnologias que thes permitam alguma vantagem competitiva.

Neste âmbito, as empresas podem encontrar na cooperação com instituições que se preocupam com a disseminação do conhecimento, a resposta aos seus anseios e/ou problemas. As instituições de Investigação e Desenvolvimento (I\&D) ligadas ou não às universidades, e outras que se preocupam com a transferência de tecnologia (TT) como forma de aumentar a competitividade da sua envolvente empresarial têm um papel fundamental, em conjunto com os decisores e autoridades governamentais. Importa saber, portanto, até que ponto estão preparadas estas Instituições para participar num processo tão complexo como o da transferência de conhecimento e de tecnologia para as empresas.

Neste contexto, é válido questionar o quanto é eficiente uma dada Instituição no que toca a tarefa de disseminar o conhecimento e a tecnologia. E se assim for, o quanto foi eficaz na prossecução daquela tarefa.

\section{A IMPORTÂNCIA DO TEMA}

A importância deste tema está diretamente ligada à constante mudança que se está a operar em todo o mundo económico e, em particular, à elevada taxa de mudança tecnológica.

É hoje perfeitamente reconhecido que o nível de vida de uma nação depende da ciência e da tecnologia. A realização do progresso de uma nação, tanto económica como culturalmente, só é possível se o desenvolvimento de recursos humanos for uma prioridade elevada. A transferência de tecnologia está no centro destes problemas e a cooperação entre Instituições ligadas à $I \& D$, e o meio empresarial 
envolvente é um dos mais importantes instrumentos para governar o processo. A ciência tornou-se assim, uma actividade humana importante, e as questões relacionadas à ciência são hoje um critério da sua maturidade [NOV91].

Este cenário de mudança/evolução da ciência e da tecnologia, olhado em conjunto com as instituições ligadas à I\&D e empresas, como actores no sistema científico e tecnológico, começa a produzir efeitos naquelas Instituições, tendo suscitado curiosidade científica no sentido de se procurar saber se os actores que participam neste processo, os mecanismos utilizados e os próprios procedimentos estão direccionados no sentido mais adequado.

Não existe uma forma padrão para verificar sistematicamente se a performance de uma Instituição, no que toca à transferência de tecnologia, é adequada, se pode melhorar ou onde se pode intervir para aumentar a sua eficiência e eficácia. Esta verificação é fulcral nos processos de melhoria. O conhecimento deste tipo de informação pode levar a uma aceleração dos processos de desenvolvimento daquelas Instituições, assim como, em relação aos governantes, instruir os programas de apoio e incentivos regionais e até nacionais na perspectiva de incentivar as iniciativas mais eficazes para as Instituições.

\section{CARACTERIZAÇÃO DO PROBLEMA}

O surgimento de instituições que promovem a interacção entre universidades e empresas tem contribuído para maximizar o processo de desenvolvimento tecnológico das empresas [COT03].

Actualmente, devidoaovertiginoso processo de inovaçãotecnológica, a cooperação entre Universidades, Institutos e outras Instituições Científicas e o tecido empresarial aparece como um importantíssimo arranjo entre instituições para promover e manter a competitividade daquelas empresas. Entretanto são inúmeros os exemplos tanto de Instituições daquele tipo, que ainda estão longe da realidade do mercado e da obtenção do êxito na investigação ou desenvolvimento em parceria, como de empresas que, mesmo consciencializadas da importância da inovação, desconhecem os meios e as formas de a buscar. 
As várias formas, as várias estratégias e locais que caracterizam os esforços para se pôr em prática processos de transferir conhecimento e/ou tecnologia são difíceis de configurar, acompanhar, e controlar ao nível da sua performance.

Como analisar e identificar sistematicamente processos de uma Instituição na sua vertente de geradora e transmissora de conhecimentos e na forma de transferir Tecnologia?

Como analisar o posicionamento daquela Instituição nos seus processos de transferência de tecnologia dado um cenário?

Como maximizar a eficiência e a eficácia das relações entre as Instituições que transferem tecnologia e o tecido Empresarial envolvente? Podemos apontar processos e mecanismos críticos que estejam pouco implementados?

Para as questões acima, o leque de variáveis que condicionam as relações de transferência de tecnologia é muito grande. Será possível caracterizar processos, identificando os níveis de implementação práticos ideais de suas etapas, em todas as suas vertentes e tipos de iteração com as Empresas?

Será possível apontar facilitadores de TT para um dado cenário, segundo uma colecção de regras para estes facilitadores de TT, que direccionem no sentido das boas práticas actuais?

\section{JUSTIFICAÇÃO PARA A ESCOLHA DO TEMA}

A nível mundial existem muitos trabalhos relacionados com a questão das relações de transferência de tecnologia. No entanto, nem todos podem ser aplicados indiscriminadamente aos vários cenários e regiões devido às suas especificidades sociais, económicas e culturais. Um aprofundamento nestes estudos permite apontar, a par da adaptação de soluções já desenvolvidas, respostas de maior eficácia na busca da excelência nos ambientes em que as várias Instituições actuam. Assim, uma ferramenta que aponte, num dado cenário, factores críticos pouco implementados ou não existentes numa Instituição de $\mathrm{I} \& D$ universitária, ou não, poderá ajudar a analisar e seleccionar acções de melhoria que aumentem a performance daquela Instituição no que toca a TT. 


\section{O TRABALHO PROPOSTO}

O domínio científico da investigação é a gestão da inovação e a gestão da transferência de conhecimento e de tecnologia, tendo como tema central as relações entre empresas e agentes de inovação que fazem da transferência de tecnologia uma das suas actividades, pelo que o objectivo foi tentar caracterizar uma ferramenta de análise adequada ao estudo daqueles agentes, enquanto disseminadores de conhecimento e as formas de maximizar a sua actuação quando tenta atender as necessidades das empresas.

Os seguintes objectivos específicos são propostos:

- evidenciar as práticas actuais que promovem a interacção nas diversas fases do processo assim como a existência de práticas transversais àqueles.

- Sugerir uma ferramenta que, utilizando os conhecimentos anteriores, possibilite a identificação de "bottlenecks" no processo e aponte um conceito de solução para a sua eliminação, apontando medidas para a melhoria de processos existentes, de forma a atender às necessidades das empresas, sem causar prejuízo à investigação e outras actividades da Instituição de I\&D.

Consideramos a hipótese de se poder construir uma ferramenta, baseada num conjunto de boas práticas, para analisar subjectivamente uma instituição de I\&D, universitária ou não, na sua vertente de transferência de tecnologia.

\section{METODOLOGIA}

Propomos um protótipo de uma ferramenta de análise que, além de identificar o que chamamos de "facilitadores" críticos (no sentido de serem os mais importantes) para aquele cenário no que toca ao relacionamento com Empresas, também pode descrever um conceito de solução baseado na reengenharia de processos e procedimentos e introdução e utilização de novos conceitos para aquela Instituição.

Para isso identificamos um conjunto de regras de boas práticas que, agrupadas nas etapas dos processos actuais de TT, reflectiram o estado 
da arte na gestão da transferência de tecnologia e ajudaram a caracterizar as Instituições de $\mathrm{I} \& \mathrm{D}$ no que toca ao relacionamento com a envolvente Empresarial.

A metodologia de investigação deste trabalho de pesquisa usou, para além da análise documental, de forma a verificar o estado da arte no que toca à gestão da transferência de tecnologia, a informação e o conhecimento adquiridos em observações participativas, com a utilização de ferramentas de consultoria de outras áreas, nomeadamente em consultorias de sistemas de informação, adaptadas a realidade das relações universidade-empresa.

Podemos dizer que a investigação é limitada ao que se faz nas instituições de I\&D da América do Norte e Europa (apesar de outras referências estarem presentes), com especial atenção às mais bem sucedidas instituições de I\&D americanas e o Department of Defense - DOD daquele país.

\section{UMA PROPOSTA DE FERRAMENTA DE ANÁLISE SUBJECTIVA}

\section{Os facilitadores e suas regras}

O nosso estudo partiu do pressuposto que existem práticas e estruturas que facilitam a interacção das instituições que promovem a transferência de tecnologia com a envolvente. Por isso reuniu-se um conjunto de facilitadores e regras que regem estes facilitadores, a partir de um estudo exaustivo das práticas actuais e o mapeamento em um "plano mestre" para TT.

\section{Os grupos de facilitadores}

A partir da figura 1, criada com o intuito de juntar todos os pontos de interesse da nossa discussão em torno do que definimos como grupos de facilitadores e as suas regras de boas práticas, mostramos o plano mestre e os actores que com ele interagem.

As 6 etapas do plano mestre para transferir tecnologia formam a base para o nosso estudo sobre os facilitadores de TT. As práticas nestas 6 etapas em conjunto com as práticas na instituição como organismo permitem aumentar ou diminuir a eficiência e eficácia dos processos de TT. Por isso 
definimos "facilitadores" para cada uma das etapas e para a instituição. Chamamos estes facilitadores de "Grupos de Facilitadores" (GF's).

As regras associadas a cada facilitador são definidas em função da sua capacidade em melhorar as características do facilitador a que corresponde, sempre com especial atenção à TT. As regras são frutos da discussão sobre o tema, da nossa experiência pessoal em gestão de empresas e consultoria, e nasceram da análise documental do que é hoje pratica corrente nas instituições que têm a TT como parte da sua missão [RES05].

Os grupos de facilitadores (GF) são os seguintes:

1. GF da instituição,

2. GF da estratégia,

3. GF da vigilância,

4. GF da promoção,

5. GF da identificação de veículos,

6. GF da transferência e

7. GF da gestão de resultados.

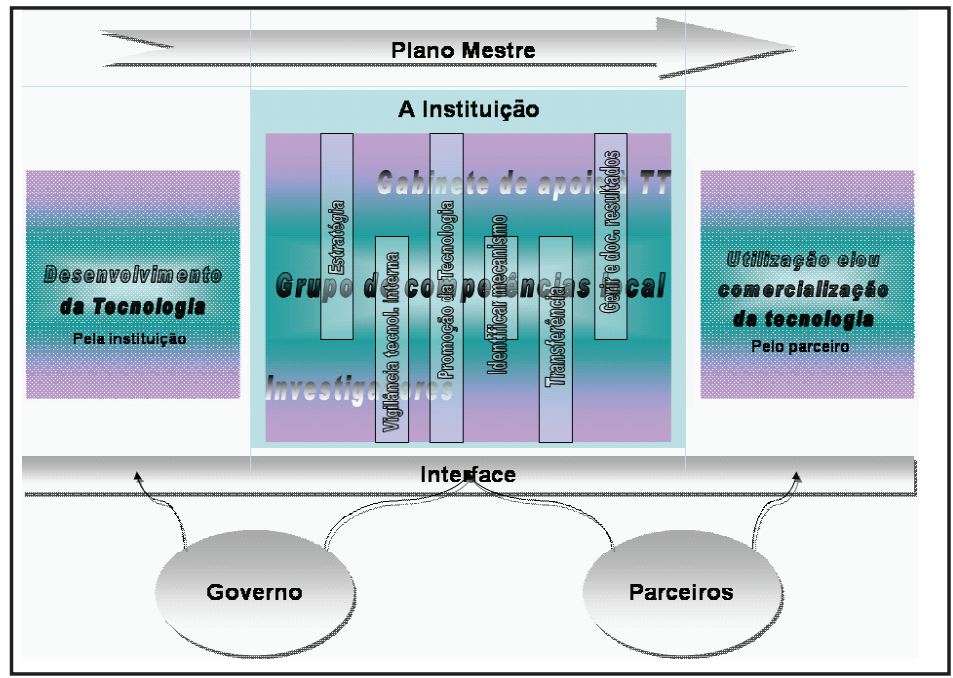

Figura 1 - O Plano Mestre com suas etapas, do ponto de vista da instituição, com as interfaces e parceiros. 
Da nossa discussão sintetizamos informações importantes para chegarmos ao nosso objectivo - verificar a possibilidade de uma ferramenta de análise poder ajudar a identificar e melhorar os processos e estruturas de uma instituição de I\&D empreendedora e voltada para a TT.

Os facilitadores não são implementados obrigatoriamente em todas as instituições de I\&D. Ou seja, existem facilitadores que não são necessários, outros que nem se quer se aplicam a uma dada situação, enquanto outros são mesmo críticos para uma instituição (e podem não o ser para outras). Um exemplo esclarecedor é o mecanismo de TT cursos de extensão/especialização que pode não ser usado numa dada instituição de $\mathrm{I} \& \mathrm{D}$, não sendo assim característico para esta, como facilitador relevante, ao mesmo tempo em que é relevante para outra que só usa este mecanismo de TT, sendo o facilitador e suas regras importantes nos seus processos e estruturas.

\section{A ESTRUTURA DA FERRAMENTA}

Podemos ver uma instituição de I\&D do ponto de vista da sua capacidade em transferir tecnologia através dos facilitadores e regras que implementa. Quanto mais facilitadores bem implementados tiver, mais preparada estará para transferir tecnologia (um facilitador está bem implementado se suas regras estão bem implementadas).

Conseguimos compilar para a nossa ferramenta 275 regras referentes a 54 facilitadores em 7 grupos de facilitadores como a seguir:

- 7 grupos de facilitadores

- GF da instituição com 6 facilitadores e 35 regras;

- GF da estratégia com 7 facilitadores e 29 regras;

- GF da vigilância com 5 facilitadores e 24 regras;

- GF da promoção com 6 facilitadores e 29 regras;

- GF da identificação de veículos com 5 facilitadores e 30 regras;

- GF da transferência com 20 facilitadores e 103 regras e

- GF da gestão de resultados com 5 facilitadores e 25 regras.

Os parágrafos anteriores definem, finalmente, o que a nossa ferramenta propôs medir. Ou seja, a análise da instituição será feita sobre as medidas dos níveis de implementação dos facilitadores relevantes para aquela instituição, a partir das suas regras. 
É importante mencionar neste ponto que a proposta de ferramenta foi sustentada em medidas subjectivas, feitas normalmente no contexto de várias entrevistas (não estruturadas) e análise documental, em que o mais importante é perceber o real entendimento dos principais actores internos engajados no subsistema da TT dentro da instituição.

\section{FACILITADORES RELEVANTES E FACILITADORES CRÍTICOS}

Devemos definir facilitadores relevantes como aqueles ligados aos processos e estruturas mais relevantes segundo a análise dos objectivos estratégicos para TT daquela instituição. São os facilitadores mais importantes, para transferir tecnologia, na perspectiva dos actores internos da instituição. $\mathrm{O}$ que não quer dizer que são os mais bem implementados!

Do que vimos até agora tiramos uma conclusão importante para o nosso trabalho: os facilitadores relevantes são os mais importantes para uma dada instituição, e quanto mais bem implementados estiverem, mais adequados estarão os processos ou estruturas correspondentes. Ao mesmo tempo vemos que ao identificarmos os facilitadores relevantes, estamos a caracterizar a instituição e os seus pontos-chave para TT segundo o nosso plano mestre. A questão que se coloca é como identificar quais são os facilitadores relevantes?

Outra questão é como identificar os facilitadores relevantes pouco implementados - os que chamaremos de facilitadores críticos? A cada uma destas fracas implementações chamaremos bottleneck.

\section{Princípios básicos}

A ferramenta que propomos deve seguir um plano sistemático para que seja realmente eficiente. Por isso vamos agora sistematizar um processo de análise que auxilie na identificação dos facilitadores relevantes no sentido daqueles que são os mais importantes na prossecução dos procedimentos de TT para a instituição em causa, segundo os seus objectivos estratégicos. A instituição, ao propor um estudo com a ferramenta em questão, mostra com clareza que está disposta a mudanças no sentido de melhorar a performance em TT. Assim, em primeiro lugar 
devemos identificar quais são os objectivos estratégicos da instituição, pois é através do processamento desta informação que vamos descobrir os facilitadores (mais) relevantes.

Com os objectivos estratégicos definidos passamos a análise actual dos processos e estruturas associados à TT, com o intuito de mapeá-los nos facilitadores do nosso plano mestre de forma a sabermos quais estão implementados na instituição.

Passamos então à medida da relevância de cada facilitador do nosso plano mestre, implementado na instituição, em relação àqueles objectivos estratégicos. Definimos então os facilitadores relevantes como o conjunto dos facilitadores encontrados na instituição cuja relevância para os objectivos estratégicos da mesma é alta.

Depois de termos os facilitadores relevantes, devemos começar a avaliar a estrutura, processos, procedimentos e ferramentas de TT, para determinar o nível de implementação dos facilitadores relevantes (os facilitadores não relevantes não necessitam avaliação por não terem, ou terem pouca importância para os objectivos estratégicos da instituição) e assim identificar os facilitadores críticos e, posteriormente através das regras críticas, os bottlenecks responsáveis pela fraca implementação serão estudados em função da ligação com os processos, estruturas, procedimentos e ferramentas de TT observados na instituição.

A identificação de um bottleneck significa que um facilitador muito importante para o alcance dos objectivos da instituição tem as suas regras pouco implementadas. Depois da identificação passamos a análise destes bottlenecks e das regras correspondentes, com tabelas e gráficos dinâmicos que permitam visualizar associações e interdependências entre os facilitadores críticos e também as influências que os aspectos da envolvente possam ter sobre os primeiros.

Em outras palavras, tentaremos desvendar quais bottlenecks são influenciados por outros. Esta informação nos permite identificar se há algum bottleneck que, ao ser modificado (melhorado por uma solução a desenhar), influencie muitos dos outros bottlenecks existentes. Se houver, obviamente será este um dos primeiros a tornar-se alvo de análise para introdução de medidas de melhorias. 
A fase seguinte é o planeamento das soluções de melhoria dos pontos fracos mais importantes, identificados através dos bottlenecks, na forma de um plano de melhoria progressivo (em etapas), onde voltamos a olhar para os processos, estruturas e ferramentas de TT que a instituição usa de forma a identificar nestas os problemas e suas causas.

\section{Procedimentos de análise}

A nossa proposta de ferramenta possibilita a identificação e proposta de solução para os bottlenecks relacionados com a gestão, os processos e as estruturas de TT, baseada nas seguintes questões essenciais:

- Quais estruturas devem ser optimizadas com vista a atingir os objectivos estratégicos da instituição como promotora da TT?

- Quais processos devem ser optimizados com vista a atingir os mesmos objectivos?

- Por onde começar?

Para responder a estas questões devemos então identificar e hierarquizar os problemas que forem encontrados na fase das entrevistas e análise documental, depois, na fase de mapeamento daqueles problemas nos facilitadores, identificar os bottlenecks correspondentes, destacando os mais prioritários e aqueles cujas soluções sejam mais fáceis e sustentáveis.

Dizemos então que as soluções sugeridas devem incidir nos bottlenecks identificados e nos processos e estruturas prioritários a melhorar.

\section{As etapas}

Na Figura 2 temos uma proposta para a sequência de etapas que julgamos adequadas à prossecução das actividades necessárias.

A análise documental, apesar de não estar presente na figura 2, tem bastante importância como fonte de informação sobre a instituição. A conjugação desta informação com o conhecimento e informação implícitos das pessoas, que tentamos obter com as entrevistas, é a base para todos os procedimentos de análise desta ferramenta. 


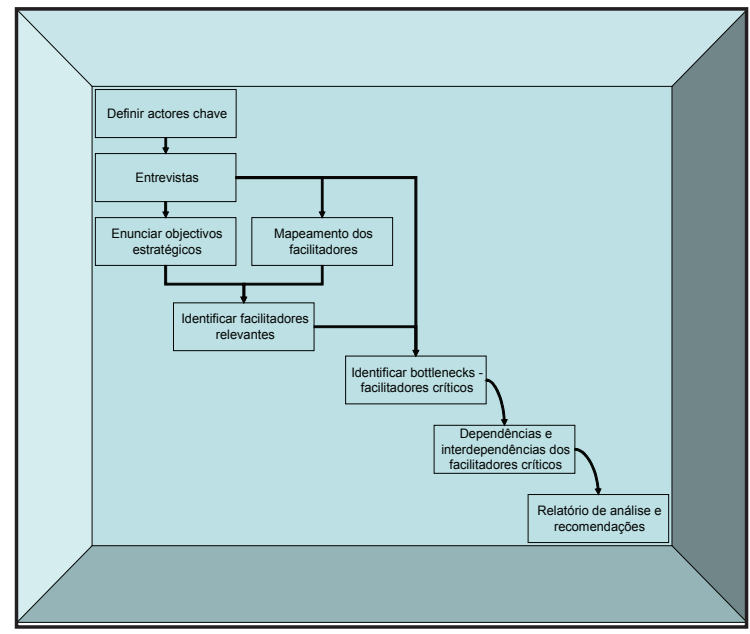

Figura 2 - Procedimentos da ferramenta de análise

\section{Definir actores-chaves}

A nossa ferramenta de análise, como já referimos, baseia-se nas entrevistas e pesquisa documental da instituição. Para termos uma visão panorâmica de todos os ângulos, devemos procurar o conhecimento e a informação que precisamos com os actores da instituição, tanto os técnicos como os administrativos, em todos os níveis, desde operacional até estratégico.

A informação a recolher a nível estratégico será, como é fácil perceber, de vital importância para definir os objectivos estratégicos para TT. A informação a recolher a nível operacional será importante para se encontrar os processos e estruturas com problemas.

A identificação dos actores-chave se faz considerando os funcionários mais relevantes, activos e que se constituam em "nós" da rede interna da instituição (continuamos a falar de TT). Actores representativos de áreas funcionais atravessadas pelos projectos de TT (horizontais), investigadores seniores participantes dos processos de TT do seu grupo local, conhecedores profundos das regras e procedimentos internos e outros actores apontados pelos próprios colegas/parceiros. 


\section{Entrevistas}

As entrevistas de análise são cruciais para a compilação da informação necessária à identificação dos objectivos estratégicos e dos bottlenecks existentes na instituição.

Os actores chave, nas entrevistas, fazem surgir os pontos de maior interesse na discussão das actividades de TT e ajudam a identificar claramente os problemas principais sentidos pelos próprios no dia-a-dia. A nível de gestão estratégica, os actores chave dão uma ideia clara dos objectivos estratégicos no que toca à TT e dos problemas que envolvem a adequação destes objectivos com relação à missão e estratégia global da instituição.

As entrevistas vão permitir o conhecimento necessário da instituição e dos processos e estruturas de TT necessários à identificação e mapeamento posterior dos facilitadores da TT.

\section{Enunciar objectivos estratégicos}

Os objectivos estratégicos para a TT podem não estar formalmente definidos. Apesar disso, devemos descobri-los e enuncia-los, pois estes são os pilares para a identificação dos facilitadores relevantes e, posteriormente, a selecção das medidas a serem adoptadas para eliminar ou atenuar os bottlenecks prioritários (melhorar a implementação dos facilitadores críticos mais importantes para os objectivos estratégicos).

\section{Mapeamento dos facilitadores}

O mapeamento é definido como a forma de relacionar os processos e estruturas identificados durante a fase das entrevistas e análise documental com os facilitadores do nosso plano mestre. Na realidade esta etapa é apenas uma pequena análise dos facilitadores que estão ou não implementados na instituição. Por isso, no final desta fase apenas estarão identificados todos os facilitadores implementados na instituição, sem informações do nível de implementação nem da relevância, que dependerá dos objectivos estratégicos.

A saída desta etapa pode ser uma tabela com três campos em que teremos de um lado os facilitadores do nosso plano mestre, ao centro a 
informação se este está presente na instituição (sim ou não) e do outro lado o local ou processo onde foi identificado o facilitador. Por exemplo, o facilitador "Planos estratégicos locais" pode estar presente porque identificamos documentos dos vários grupos locais onde se definem estes planos.

No exemplo anterior, apesar de estar presente, não significa que é relevante para os objectivos estratégicos da instituição no que toca a TT, nem que está bem implementado (suas regras segundo o nosso plano mestre ainda não foram analisadas e não se sabe se estão bem implementadas).

\section{Identificar facilitadores relevantes}

A identificação dos facilitadores relevantes é feita após o término da análise documental e das entrevistas, quando já temos uma visão detalhada o suficiente para saber, dos processos e estruturas de TT identificados, dos facilitadores mapeados e dos objectivos estratégicos, que facilitadores identificados na instituição são importantes para alcançar as metas que os objectivos estratégicos preconizam.

A ferramenta utiliza uma forma gráfica para mostrar as relevâncias dos facilitadores em referência aos objectivos estratégicos definidos para TT na instituição, onde se usa a escala de Likert com apenas três níveis (1-para pouco relevante, 3-para relevante e 5-para muito relevante) para termos distâncias relativas maiores nos gráficos. A figura 3 mostra como se identificam graficamente os facilitadores relevantes, cujo grau de relevância para ser considerado nas próximas fases deve ser discutido com os actores chave da gestão estratégica.

A referência ao grau de relevância para que um facilitador seja considerado "facilitador relevante" é importante por ser uma decisão estratégica. Para facilitar e simplificar o processo, sugerimos considerar apenas os facilitadores com grau de relevância máximo ( 5 na nossa escala, que corresponde a $100 \%$ na figura 3), visto que a nossa proposta de ferramenta visa à focalização com soluções simples e rápidas, inseridas num plano de acções sequenciais que visa a correcção dos bottlenecks hierarquicamente mais importantes em primeiro. 
uma ferramenta de análise...

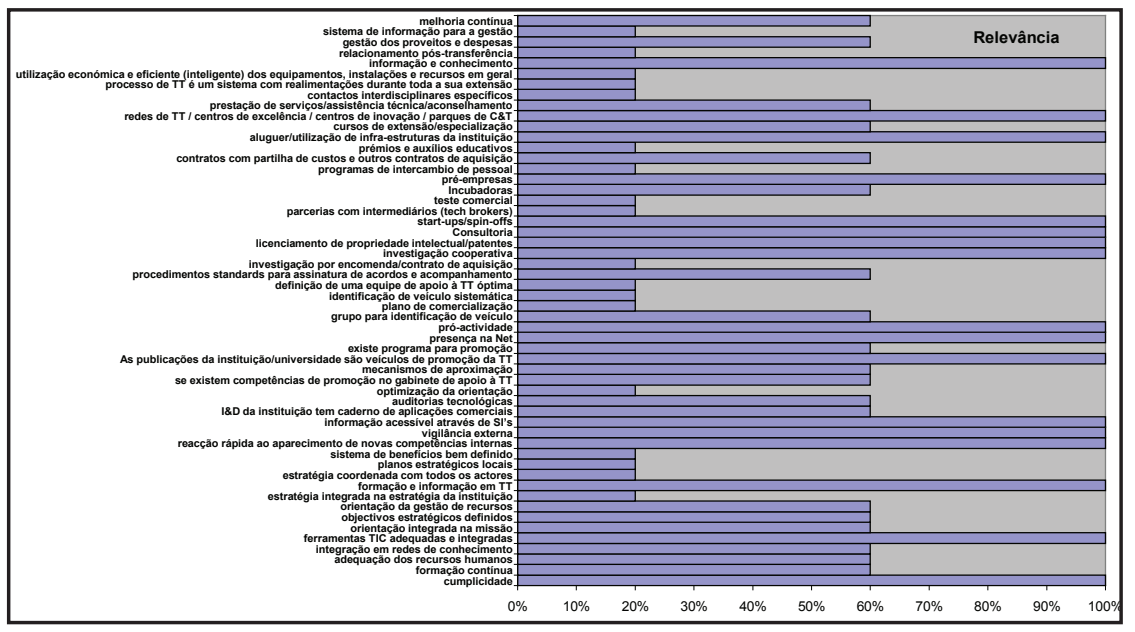

Figura 3 - Relevância dos facilitadores (um exemplo)

\section{Identificar bottlenecks - Os facilitadores críticos}

Esta é a fase em que vamos analisar as regras correspondentes aos facilitadores identificados como relevantes.

Os especialistas que aplicam esta ferramenta, neste ponto, juntam-se para analisar a instituição à luz do nosso plano mestre. É a fase de verificar, para cada facilitador presente e relevante para a instituição, as regras que são importantes, segundo os objectivos estratégicos, e os seus níveis de implementação.

Ou seja, para cada facilitador relevante vamos estudar o grau de importância das suas regras e o grau de implementação destas. Por exemplo, para o facilitador "reacção rápida ao aparecimento de novas competências internas" vamos definir o grau de importância (ou relevância) de cada regra para o seu facilitador ser eficaz e montamos, como na figura 4, uma folha de cálculo que calcule o nível de implementação do facilitador em função dos níveis de implementação de suas regras como uma média ponderada pelas relevâncias das suas próprias regras.

Assim, conforme se vê na figura 4, o grau de implementação do facilitador "reacção rápida ao aparecimento de novas competências 
internas" é de 1,5 na escala de Likert de 1 a 5, em que 1 significa pouco implementado e 5 significa muito implementado. $\mathrm{O}$ cálculo realizado foi:

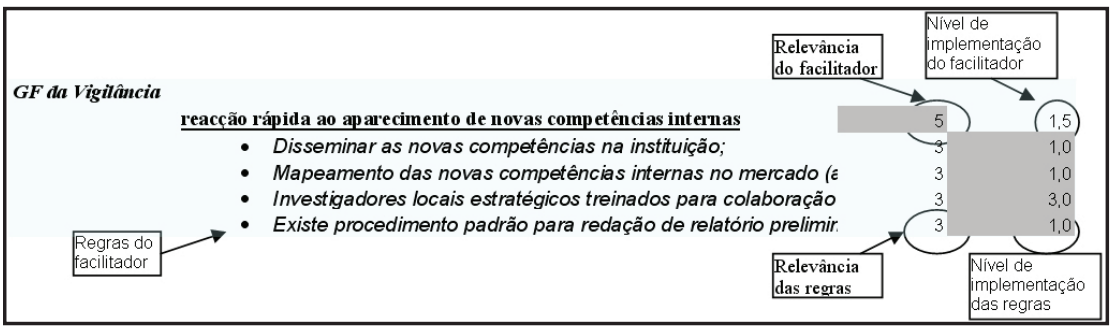

Figura 4 - Exemplo da folha de cálculo dos facilitadores com os níveis de relevância e de implementação das suas regras.

$$
\frac{\sum \text { relev } . \times \text { implement. }}{\sum \text { relev } .}=\frac{3 \times 1+3 \times 1+3 \times 3+3 \times 1}{3+3+3+3}=1,5
$$

Este valor em percentagem é equivalente a $\frac{1,5 \times 100}{5}=30 \%$, o que é pouco e caracteriza este facilitador como crítico (um bottleneck foi encontrado).

Temos aqui uma questão pertinente. Qual deverá ser o limite de implementação mínimo a partir do qual um facilitador deva ser considerado crítico? No caso acima, se considerarmos o limite de 35\%, o facilitador é crítico, mas se considerarmos o limite de $25 \%$, o facilitador já não será considerado crítico (foi considerado implementado a 30\%), fazendo com que este facilitador não seja mais considerado, daqui para a frente, no estudo dos facilitadores críticos.

Sugerimos que, sendo este limite subjectivo, se utilize um valor inversamente proporcional ao nível de focagem que se queira dar ao estudo.

Da folha de cálculo sugerida anteriormente podemos agora retirar os facilitadores relevantes com os seus graus de implementação conforme a figura 5 que já mostra a informação de uma forma particularmente vantajosa para que possamos tirar o conhecimento necessário às fases seguintes. 
uma ferramenta de análise...

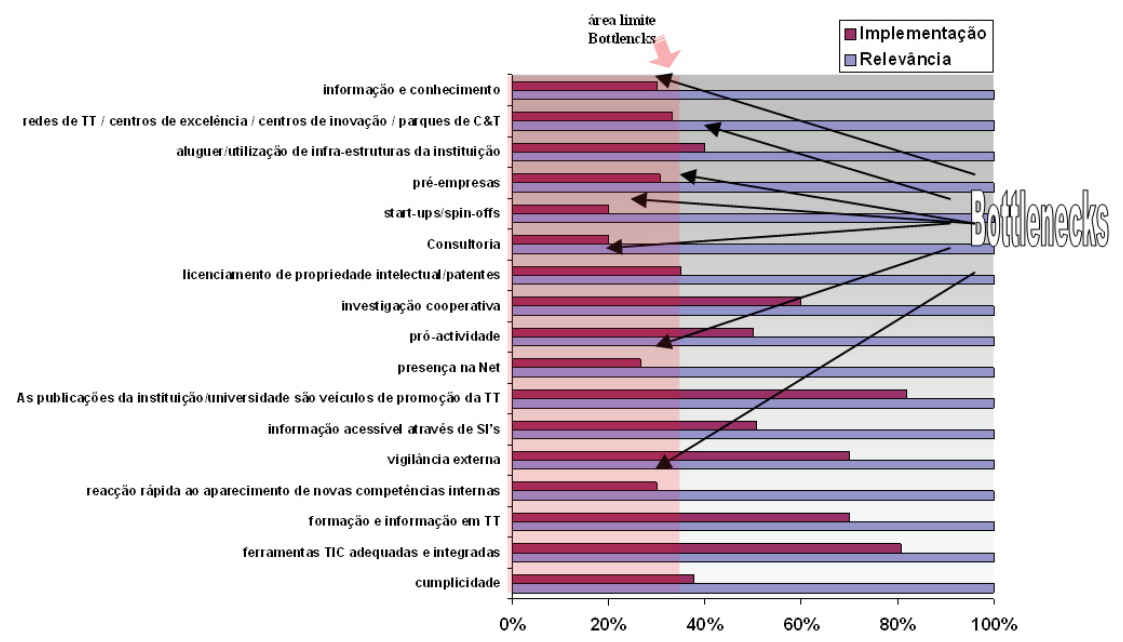

Figura 5 - Exemplo do gráfico de facilitadores relevantes e suas implementações. Deste gráfico retiramos os facilitadores críticos.

Apontamos no gráfico os bottlenecks encontrados para o limite de implementação mínimo de 35\%.

Os 7 facilitadores críticos apontados no exemplo da figura 5 passam a ser agora o centro das atenções. São estes facilitadores críticos, onde os bottlenecks foram encontrados, que são analisados entre si (interdependências) e em relação à envolvente na etapa seguinte.

As regras mais críticas destes facilitadores críticos serão analisadas com maior atenção nas fases seguintes. São elas as responsáveis, em princípio, pelos bottlenecks.

\section{Dependências e interdependências dos facilitadores críticos}

A matriz da figura 6 tem por objectivo medir o grau de autocorrelação entre os próprios facilitadores críticos, e também a influência dos factores externos sobre os mesmos facilitadores.

Esta etapa inicia-se com o preenchimento das linhas e colunas da matriz com os facilitadores críticos identificados na etapa anterior. 


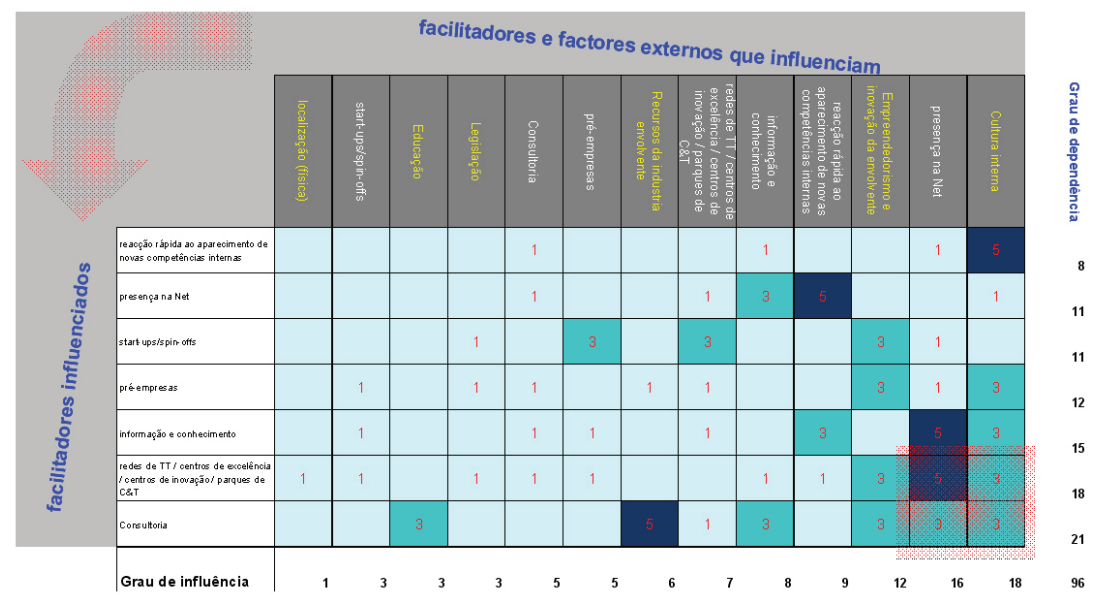

Figura 6 - Tabela de correlações - influências e dependências entre facilitadores e factores externos (exemplo de preenchimento com valores). Facilitadores classificados análise mais focada.

O passo seguinte é o preenchimento do corpo da matriz com valores que reflectem as influências dos facilitadores das colunas nos mesmos facilitadores, agora nas linhas horizontais (títulos à esquerda). Depois os valores que reflectem as influências de factores externos, que estão listados nas colunas da direita, também nos mesmos facilitadores horizontais. Conforme podemos verificar na figura 6 , para o exemplo anterior, os somatórios das influências são mostrados a direita e em baixo da matriz.

O nosso intuito é utilizar esta informação para descobrir quais são os facilitadores (e factores externos) que mais influenciam outros, e quais são os que mais são influenciados (mais dependentes - sensíveis).

Com esse objectivo e após o preenchimento da matriz, fazemos duas operações de ordenamento da matriz. A primeira será ordenar a matriz na sequência dos totais das influências dados pela última linha da matriz. Ou seja ordenar a matriz pelos valores da última linha (grau de influência).

Depois, ordenar a matriz na sequência do grau de dependência total dos facilitadores. Ou seja, ordenar a matriz pelos valores da última coluna. Com isso, apesar de ser a mesma matriz, temos uma visão mais adequada 
com a concentração dos facilitadores críticos que mais influenciam e os mais sensíveis no canto inferior direito.

Agora, se quisermos ser muito precisos na escolha dos facilitadores a analisar numa próxima fase com vista a melhorias, usamos os facilitadores mais a direita e mais abaixo. Quanto mais abrangentes (ou generalistas, se preferirmos a expressão) quisermos ser, maior deve ser a área do rectângulo que abrange os valores totais mais altos na matriz conforme a figura 6 .

Para um melhor esclarecimento vamos discutir o exemplo da figura 6 , onde os facilitadores críticos que mais influenciam são a "presença na net" e a "reacção rápida a novas competências internas".

Neste exemplo verificamos que há alguns factores externos (ao subsistema de TT) que também devem ser considerados como problemas para reflexão. São eles a "cultura interna" e o "empreendedorismo e inovação da envolvente".

Pelo lado dos facilitadores mais "sensíveis" podemos notar no exemplo que os facilitadores "consultoria”, "redes de TT e etc.”, “informação e conhecimento" e "pré-empresas” são, em ordem decrescente, os mais influenciados.

Com relação à focagem na análise, se diminuirmos o rectângulo da direita e em baixo como na matriz da figura 7 , direccionamos a nossa atenção a um número menor de facilitadores e factores externos influenciadores. $\mathrm{Na}$ figura 6 vemos esta focalização feita. Nesse caso o facilitador que mais influência tem sobre os outros, a "presença na net", e o factor "cultura interna” serão o foco da análise posterior em conjunto com os 2 facilitadores mais sensíveis.

O próximo passo é, a partir da análise dos facilitadores mais críticos identificados na matriz anterior e do conhecimento dos constrangimentos externos mais importantes, mapearmos de volta os facilitadores, que julgamos mais importantes serem reavaliados, nos processos, estruturas e procedimentos de TT da instituição.

\section{Relatório de análise e recomendações}

O relatório de análise e recomendações é o documento que apresenta os resultados da análise feita à instituição de $\mathrm{I} \& \mathrm{D}$ sobre seus processos, 
procedimentos e estruturas envolvidos na TT. Põe em evidência os facilitadores críticos e factores externos mais importantes mostrando os resultados da análise de forma gráfica - figura 5 e figura 6 .

Além disso, propõe recomendações, baseadas na reengenharia dos processos, nos procedimentos e melhorias nas infra-estruturas, que mais influenciam os facilitadores críticos mais importantes (focagem), sem esquecer que os factores externos de influência devem ser neutralizados - ou pelo menos atenuados, segundo os resultados espelhados na matriz da figura 6. As recomendações de acções de resolução devem ser as economicamente mais relevantes segundo a razão custo/benefício.

O material de análise, fonte de informação de que dispomos, é o resultado das entrevistas, visitas de estudo aos locais de maior interesse (observação), actas das reuniões (da reunião de remapeamento principalmente) e da documentação disponível na instituição.

O documento deve explicitar os processos, procedimentos e estruturas de TT da instituição relacionados com as regras críticas mais importantes segundo os resultados da reunião de remapeamento.

Com o intuito de descrever este relatório mostramos na figura 7 uma proposta de índice para o documento. Deve-se observar que, apesar de não ser nosso intuito criar um modelo para o documento, mostramos os pontos que julgamos importantes estarem presentes.

\section{CONCLUSÕES E NOTAS FINAIS}

No estudo observamos que a transferência de tecnologia das universidades e instituições de I\&D é uma realidade já há muito tempo. Entretanto, a forma com que se tem implementado na prática tem sido um tanto quanto desordenada e sem estruturação.

Mesmo assim temos visto muitos casos de sucesso, algumas regiões de excelência, outras com grandes capacidades para assimilar o conhecimento gerado nas instituições nelas instaladas e muitas empresas com capacidades latentes para inovar e relacionarem-se com as instituições de I\&D mais próximas.

Concluímos desta forma que a necessidade de organizar e sistematizar as relações entre as instituições que geram conhecimento e os potenciais 


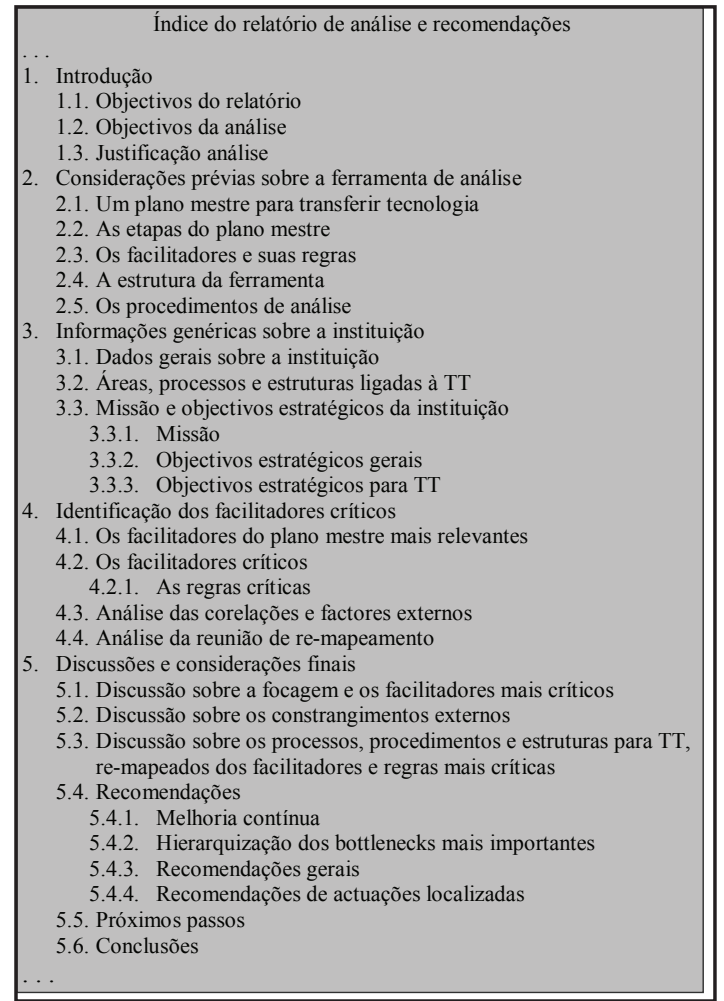

Figura 7 - Proposta de estrutura do relatório de análise e recomendações

clientes deste conhecimento se mostra patente na forma incipiente e esporádica dos casos pontuais que se vêm na prática em geral.

Em nossa opinião, uma instituição que ponha em prática um plano bem estruturado, com processos bem definidos, sistemáticos e utilizando todo 0 potencial das estruturas disponíveis da organização, consegue disseminar o seu conhecimento de uma forma muito mais constante, enriquecendo assim a sociedade em que se insere.

O plano mestre não tem a pretensão de ser um modelo. Nasceu da sua necessidade e importância para a ferramenta de análise. Os alicerces 
deste plano foram as boas práticas actuais nos processos de transferência de tecnologia. Além disso, foi criado a pensar na rentabilização das estruturas e infra-estruturas presentes nas instituições que passariam a participar naqueles processos transversais. Queremos dizer com isso que a necessidade de criar um plano mestre veio da constatação de que, para analisar sistematicamente uma instituição, na sua vertente de disseminadora de conhecimento, era necessário mapear os processos desta num conjunto de boas práticas actuais, de forma a identificar onde estão os Bottlenecks (facilitadores críticos pouco ou não implementados).

$\mathrm{O}$ nosso estudo partiu do pressuposto que existiam práticas e estruturas que facilitavam a interacção das instituições que promovem a transferência de tecnologia com a envolvente. Assim, construímos um Plano Mestre e, com base neste, reunimos um conjunto de facilitadores, cada um com as suas regras. Conseguimos compilar para a nossa ferramenta 275 regras referentes a 54 facilitadores em 7 grupos de facilitadores.

Podemos ver uma instituição de I\&D, do ponto de vista da sua capacidade em transferir tecnologia, através dos facilitadores e regras que implementa. Quanto mais facilitadores bem implementados tiver, mais preparada estará para transferir tecnologia (um facilitador está bem implementado se suas regras estão bem implementadas).

Concluindo, a ferramenta proposta pretende ser uma forma padrão para verificar sistematicamente se a performance de uma Instituição, no que toca à transferência de tecnologia é adequada, se pode melhorar ou onde se pode intervir para aumentar a sua eficiência e eficácia. Esta verificação é fulcral nos processos de melhoria. As vantagens da utilização desta ferramenta para análise preliminar com vista a melhorias nos procedimentos e estruturas utilizados para transferir tecnologia são, em primeiro lugar, soluções focadas nos bottlenecks e nos processos e estruturas críticas mais importantes, possibilitando investimentos menores e/ou em etapas. Em segundo lugar a identificação dos problemas mais rapidamente através dos facilitadores mais críticos menos implementados. Em terceiro lugar, um único estudo deixa as várias medidas de optimização necessárias de uma forma hierarquizada para serem implementadas em etapas. 
Portanto, conseguimos mostrar que é possível utilizar uma ferramenta como a mencionada na hipótese inicial, cujos objectivos garantem soluções para os problemas que queríamos mostrar serem possíveis de se ultrapassar.

Esta ferramenta é a base para a criação de uma metodologia que tenha por objectivo suportar eficientemente o redesign e a especificação de novos procedimentos de TT baseados no trabalho em equipa que melhorem os processos interdepartamentais de uma universidade ou instituição de I\&D. Não foi nosso intuito criar modelos, descrever as melhores práticas em TT nem inventar métodos de análises de empresas. Por isso, consideramos o nosso trabalho como uma introdução ao que poderíamos fazer no futuro.

\section{REFERÊNCIAS}

[ABE04] APG Aberdeen Proving Ground. Science and Technology Board - Tech Transfer Mechanisms. http://stb.apg.army.mil/mechanisms/Transfer.htm (acesso em 2004).

[AIR02] Air Force Research Laboratory. Air Force Technology Transfer Handbook. http://www.afrl.af.mil . (2002).

[AUT02]AUTM - Association of University Technology Managers. AUTM Licensing Survey: FY 2002 e AUTM Journal, volume XIV 2002.

[COT03] Cotec, Fundación Cotec para la innovación tecnológica. "Nuevos mecanismos de transferencia de tecnología - Debilidades y oportunidades del Sistema Español de Transferencia de Tecnología. Encuentros Empresariales COTEC”. Gijón, 2003.

[FLC04] Federal Laboratory Consortium for Technology Transfer. Technology Transfer Desk Reference. 2004. http://www.federallabs.org

[LOU02] Universidade Católica de Louvain. Research and Development Liaisons Office. http://www.ucl.ac.be/intro-en.html (acesso em 2002).

[NOV91] Novozhilov, Y. V. University-Industry Cooperation in Eastern Europe: The experience of some advanced Universities in the USSR. International Journal of Technology Management, vol. 6, $n^{0}$ 5/6. 1991 .

[RES05] Resende, David. Diz, Henrique. Transferência de tecnologia - as práticas actuais e uma ferramenta para análise subjectiva das instituições. Tese de Mestrado 


$$
\text { revista tecnologia e sociedade }
$$

realizada sob orientação científica do Professor Doutor Henrique Diz. Universidade de Aveiro - DEGEI, Aveiro 2005. 